\title{
Comentario a «Asociación de la rigidez peneana nocturna con testosterona, síndrome metabólico y otras variables: un estudio piloto prospectivo transversal»
}

\section{Comment to "Association of nocturnal penile rigidity with testosterone, metabolic syndrome, and other variables: a prospective cross-sectional pilot study"'}

\section{R. Vela-Navarrete}

Servicio de Urología, Fundación Jiménez Díaz, Madrid, España

El extraordinario atractivo profesional y científico del padecimiento exclusivamente masculino (salud de género), que conocemos con el nombre de disfunción eréctil, ha motivado el interés de numerosas especialidades; la mayoría de ellas tiene, sin duda alguna, conocimientos complementarios para entender mejor y tratar este proceso, más aun desde que se conoce que la disfunción eréctil suele venir «acompañada» muy especialmente por factores patológicos vasculares. Es ahora, aparentemente, un concepto progresista inventar síndromes muy alejados de los postulados marcados por la Medicina anatomo-clínica tradicional: un síndrome clínico definido y concreto, a veces referido exclusivamente a un grupo poblacional o un género, teniendo como fundamento una lesión anatómica también específica y concreta. El concepto de síndrome metabólico nació alrededor de un tipo de obesidad singular asociada a hipertensión, trastornos del metabolismo lipídico y diabetes tipo 2 o resistente a la insulina. Progresivamente, este síndrome ha ido extendiéndose $\mathrm{y}$, finalmente, ha incluido, gracias a iniciativas originadas en la esfera de la Endocrinología 1 , la androgenodeficiencia y la disfunción eréctil. Cuando se analiza la evolución del síndrome metabólico desde la perspectiva de la clínica urológica se comprueba que el eje

Véase contenido relacionado en DOI:

10.1016/j.acuro.2011.03.014.

Correo electrónico: RVela@fjd.es fundamental del mismo, la diabetes tipo 2, es casi de prevalencia exclusiva masculina, y que el resto de los datos son mucho más comunes en el varón que en la mujer, para concluir que, aparentemente, estamos hablando del envejecimiento masculino ${ }^{2}$. Choca aquí frontalmente el compromiso actual de la Urología y su programa de salud de género con otras iniciativas profesionales, como aquellas que van ampliando progresivamente las fronteras del síndrome metabólico.

Hasta ahora la práctica urológica habitual ha tratado al varón y, especialmente, al varón añoso, digamos por encima de los 50 años, «a trozos», por órganos: en unos se ocuparía de su prostatismo, en otros del potencial riesgo de un PSA elevado o de trastornos de la erección o eyaculación, etc. Rara vez la práctica urológica diaria mira al enfermo cabalmente, integralmente, al menos preguntando por su tensión arterial y medicamentos hipotensores o antihipertensivos, diabetes, antiarrítmicos, peso, índice de masa corporal (IMC), etc. Tan negativa ha sido esta visión general del enfermo urológico que alguien ha acuñado el concepto de «el enfermo oculto detrás de la próstata» y en contraposición, y ya desde una perspectiva moderna, progresista, de salud de género, la de reconvertir al urólogo en generalista del varón ${ }^{3}$. Y es ahora cuando este proyecto de futuro comienza a ser reconocido como piedra angular de la práctica médica urológica futura, cuando aquellos urólogos más interesados por una visión integral del paciente, representados por la práctica andrológica, comienzan a reconocer las 
coincidencias entre el síndrome metabólico y el síndrome urológico que define al varón añoso ${ }^{2}$.

El trabajo de los Dres. Rajmil et al ${ }^{4}$ sobre la asociación de la rigidez peneana nocturna con los niveles séricos de testosterona, síndrome metabólico y otras variables es el mejor exponente de esta transición que advierte sobre la salud de género y sobre el interés cada día mayor de la Urología por la salud de género y, especialmente, por la salud de género del varón añoso. Este trabajo toma como punto de análisis de correlaciones y comorbilidades 234 varones que consultan por trastornos de la función eréctil, y que son sometidos a un estudio de existencia e intensidad de la rigidez de las erecciones nocturnas (NPTR) con el instrumento apropiado (Rigiscan). Estos hallazgos son comparados con los niveles séricos de testosterona, síndrome metabólico e IMC. Los hallazgos concuerdan con lo esperado y previamente observado en la literatura, pero hay varios puntos extraordinariamente importantes que, como indican los mismos autores, carecen de fortaleza estadística por lo reducido de la muestra. Efectivamente, hay una limitación en este análisis, fundamentalmente relacionada con la edad de la población incluida, entre 18 y 85 años, un grupo poblacional absolutamente heterogéneo. Con certeza, si la totalidad de la población incluida hubiera tenido más de 50 años las debilidades del análisis estadístico hubieran sido superadas, con firmes evidencias de que existen relaciones entre edad y disfunción eréctil, niveles de testosterona, diabetes tipo 2, hipertensión, marcadores de disfunción endotelial, inflamatorios, etc ${ }^{5}$. En definitiva, este trabajo debe ser valorado no sólo desde la visión tradicional de su calidad científica, sino desde la nueva perspectiva que invita al urólogo, en general, y más aún al específicamente dedicado a la Andrología, a considerar al paciente urológico de manera integral, aproximándose en su quehacer diario cada vez más a la propuesta del generalista del varón.

\section{Bibliografía}

1. Zitzman M. Testosterone deficiency, insulin resistance and the metabolic syndrome. Nat Rev Endocrinol. 2009;5:67381.

2. Vela Navarrete R. El síndrome urológico del envejecimiento masculino: una visión integral e integradora. Tiempos Médicos. 2010;667:3-5.

3. Kirby RS, Kirby MG, Farah RN. Men's Health. Oxford: ISIS Medical Media; 1999.

4. Rajmil $O$, Fernández $M$, Blasco A, Arrús JA, Montañés $R$, Rodríguez-Espinosa J. Asociación de la rigidez peneana nocturna con testosterona, síndrome metabólico y otras variables: un estudio piloto prospectivo transversal. Actas Urol Esp. 2011;35:459-67.

5. Vela Navarrete R, García Cardoso JV, Pardo Montero M, Jiménez Mateos-Cáceres P, Lopez Farré A. Testosterona, función endotelial, salud cardiovascular y androgenodeficiencia del varón añoso. Arch Esp Urol. 2009;62:173-8. 